\title{
Observation of magnetic field generation via the Weibel instability in interpenetrating plasma flows
}

\author{
C. M. Huntington ${ }^{1 \star}$, F. Fiuza ${ }^{1}$, J. S. Ross ${ }^{1}$, A. B. Zylstra ${ }^{2}$, R. P. Drake ${ }^{3}$, D. H. Froula ${ }^{4}$, G. Gregori ${ }^{5}$, \\ N. L. Kugland ${ }^{6}$, C. C. Kuranz ${ }^{3}$, M. C. Levy ${ }^{1}$, C. K. Li ${ }^{2}$, J. Meinecke ${ }^{5}$, T. Morita ${ }^{7}$, R. Petrasso ${ }^{2}$, C. Plechaty ${ }^{1}$, \\ B. A. Remington ${ }^{1}$, D. D. Ryutov ${ }^{1}$, Y. Sakawa7 , A. Spitkovsky ${ }^{8}$, H. Takabe ${ }^{7}$ and H.-S. Park ${ }^{1}$
}

\begin{abstract}
Collisionless shocks can be produced as a result of strong magnetic fields in a plasma flow, and therefore are common in many astrophysical systems. The Weibel instability is one candidate mechanism for the generation of sufficiently strong fields to create a collisionless shock. Despite their crucial role in astrophysical systems, observation of the magnetic fields produced by Weibel instabilities in experiments has been challenging. Using a proton probe to directly image electromagnetic fields, we present evidence of Weibelgenerated magnetic fields that grow in opposing, initially unmagnetized plasma flows from laser-driven laboratory experiments. Three-dimensional particle-in-cell simulations reveal that the instability efficiently extracts energy from the plasma flows, and that the self-generated magnetic energy reaches a few percent of the total energy in the system. This result demonstrates an experimental platform suitable for the investigation of a wide range of astrophysical phenomena, including collisionless shock formation in supernova remnants, large-scale magnetic field amplification, and the radiation signature from gamma-ray bursts.
\end{abstract}

The magnetic fields required for collisionless shock formation in astrophysical systems may either be initially present, for example in supernova remnants or young galaxies ${ }^{1}$, or they may be selfgenerated in systems such as gamma-ray bursts (GRBs; ref. 2). In the case of GRB outflows, the intense magnetic fields are greater than those which can be seeded by the GRB progenitor or produced by misaligned density and temperature gradients (the Biermannbattery effect) $)^{3,4}$. It has long been known that instabilities can generate strong magnetic fields, even in the absence of seed fields. Weibel considered the development of an electromagnetic instability driven by the electron velocity anisotropy in a background of resting ions $s^{5}$. The signature of the instability is a pattern of current filaments stretched along the axis of symmetry of the electron motion. The process is quite general, and subsequent work has shown that such instabilities can be excited in both non-relativistic and relativistic shocks. This general nature makes the Weibel instability common in astrophysical systems ${ }^{6-8}$. The instability provides a mechanism by which the electromagnetic turbulence associated with the formation of collisionless shocks is fed by the flow anisotropy of the protons (and ions) stochastically reflecting off of the shock ${ }^{9-11}$, and leading ultimately to strong particle acceleration in GRB's (ref. 12).
The importance of Weibel instabilities in astrophysical systems makes laboratory experiments that can access the collisionless plasma regime particularly compelling, although so far experiments have been limited to collisional systems (where Weibel instability growth is limited by collisional dissipation ${ }^{13}$ ) or those where the initial plasma conditions are not well characterized ${ }^{14,15}$. Reaching the collisionless regime also permits the instability dynamics to be described by dimensionless parameters and scaled between laboratory and astrophysical systems ${ }^{16}$. In the collisionless regime, the scaling is remarkably simple and allows one to predict the parameters of the unstable modes and the shocks (should they be formed) on the basis of laboratory measurements and the astrophysical 'input' parameters, the density and velocity of the flows.

In experiments performed at the Omega Laser Facility ${ }^{17}$, we directly image the magnetic fields associated with the Weibel instability in well-characterized, counter-streaming plasma flows in the collisionless plasma regime ${ }^{18}$. The flows were established by laser ablation of opposing foils, as shown in Fig. 1. The foils were oriented opposite each other and irradiated simultaneously, such that the expanding plasma flows interacted near the midplane between the foils ${ }^{19-21}$. The plasma conditions in this geometry have been previously measured under identical conditions with Thomson scattering ${ }^{18}$. When only a single foil was used, the plasma flow velocity $v$ was measured to be $1,000-2,000 \mathrm{~km} \mathrm{~s}^{-1}$, with an electron density $\left(n_{\mathrm{e}}\right) \approx 5 \times 10^{18} \mathrm{~cm}^{-3}$ and electron and ion temperatures $\left(T_{\mathrm{e}}, T_{\mathrm{i}}\right)$ less than $200 \mathrm{eV}$.

When two opposing foils are used, as in the present work, the plasma density in the counter-propagating flows increased by the anticipated factor of two, whereas the electron and ion temperatures increased rapidly owing to a combination of collisional electron heating and ion two-stream instability ${ }^{20}$. The ion instability quickly stabilizes as the electron and ion temperatures equilibrate, which was observed to occur near $1 \mathrm{keV}$. The ions remain directed throughout the process, allowing competing instabilities, including the Weibel instability, to grow from the energy supplied by the flows ${ }^{22}$.

Magnetic fields are detected in this experiment using proton imaging. An isotropically emitting proton source is generated by the implosion of a capsule filled with $\mathrm{D}-{ }^{3} \mathrm{He}$, producing protons at $3 \mathrm{MeV}$ (from $\mathrm{D}-\mathrm{D}$ reactions) and at $14.7 \mathrm{MeV}$ (from $\mathrm{D}-{ }^{3} \mathrm{He}$

\footnotetext{
${ }^{1}$ Lawrence Livermore National Laboratory, Livermore, California 94550, USA. ${ }^{2}$ Massachusetts Institute of Technology, Cambridge, Massachusetts 02139 , USA. ${ }^{3}$ Department of Atmospheric, Oceanic, and Space Sciences, University of Michigan, Ann Arbor, Michigan 48109, USA. ${ }^{4}$ Physics Department and Laboratory for Laser Energetics, University of Rochester, Rochester, New York 14636, USA. ${ }^{5}$ Department of Physics, University of Oxford, Parks Road, Oxford OX1 3PU, UK. ${ }^{6}$ Lam Research Corporation, 4400 Cushing Parkway, Fremont, California 94538, USA. ${ }^{7}$ Institute of Laser Engineering, Osaka University, Osaka 565-0871, Japan. ${ }^{8}$ Department of Astrophysical Sciences, Princeton University, Princeton, New Jersey 08544, USA. *e-mail: huntington4@llnl.gov
} 


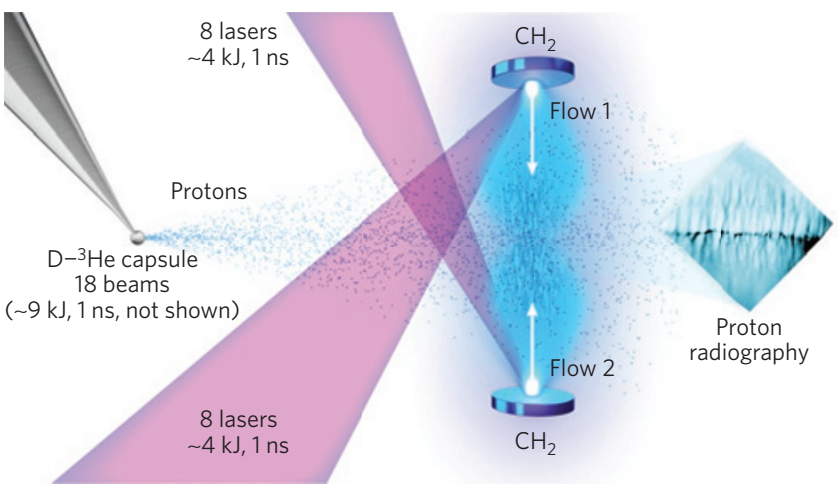

Figure 1 | Experimental configuration to generate opposing plasma flows probed by $\mathrm{D}-{ }^{3} \mathrm{He}$ protons. The experiment consists of a pair of $\left(\mathrm{CH}_{2}\right)$ plastic foils of diameter $2 \mathrm{~mm}$ and thickness $500 \mu \mathrm{m}$, oriented face-on and separated by $8 \mathrm{~mm}$. Each was irradiated with eight overlapped laser beams, delivering $\sim 4 \mathrm{~kJ}$ of $351 \mathrm{~nm}$ laser energy in a $1 \mathrm{~ns}$ square pulse. Distributed phase plates were used to produce super-Gaussian laser spots with focal spot diameters of $250 \mu \mathrm{m}$ on the target surface. After a delay, the proton probe was created by laser-compressing a thin-walled $\mathrm{SiO}_{2}$ capsule. The capsule was filled with a 1:1 mixture of deuterium (D) and ${ }^{3}$ helium $\left({ }^{3} \mathrm{He}\right)$ at a total pressure of $18 \mathrm{~atm}$. At peak compression $\left(10^{23} \mathrm{~cm}^{-3}\right)$ protons are produced quasi-isotropically at energies of 3.0 and $14.7 \mathrm{MeV}$. The protons were detected using a CR39 nuclear track detector positioned on the midplane of the $\mathrm{CH}_{2}$ target foils, such that the protons traverse the central interaction region as shown.

reactions; see Supplementary Information for additional details on proton imaging). The protons that pass through the plasma interaction region are deflected by the electric and magnetic fields in the system, and are recorded using a CR39 nuclear track detector at a magnification of approximately 30 . There are several important features in the proton radiography data, which was taken at three different times during the interaction of the flows, and are shown on the top two rows of Fig. 2. First, oriented along the flow direction is a pattern of filamentary structures, consistent with Weibel filamentation in the counter-propagating flows. These features develop strongly between 3 and 4 ns after the start of the drive laser pulse and grow to lengths $>1 \mathrm{~mm}$ along the direction of the flow. The filamentary structure is clear in both 3.0 and $14.7 \mathrm{MeV}$ proton images, and extends relatively uniformly for several $\mathrm{mm}$ across the plasma flow, occupying a total volume of several $\mathrm{mm}^{3}$. The similarity in the observed features and relative contrast between the $3 \mathrm{MeV}$ and $14.7 \mathrm{MeV}$ radiographs indicate that proton deflections were produced by magnetic fields (see Supplementary Information for further discussion).

In addition to the filaments, horizontal 'plate' features are seen near the midplane of the drive plasmas. These large-scale magnetic features have been observed in previous experiments with similar geometries $^{14,19}$, and are understood to be the result of Biermannbattery-generated magnetic fields ${ }^{23}$. These fields are created at the target surface during the laser ablation and form a loop around the expanding plasma flow ${ }^{24,25}$. The Biermann fields are frozen in the flow, following the effective electron trajectory to the midplane, where the longitudinal electron velocity from the two flows is cancelled. The magnetic fields cannot readily cross the midplane, and expand transversely, leading to the formation of characteristic plates $^{23}$. Asymmetry between the top and bottom plates in the data is related to slight non-uniformities in the flows, including differences in laser energy deposition on the two foils and tilts in their orientation relative to the proton probe.

To better understand both the Weibel and Biermann-batterygenerated magnetic fields in the experiment we have conducted detailed 3-dimensional particle-in-cell (3D PIC) simulations with

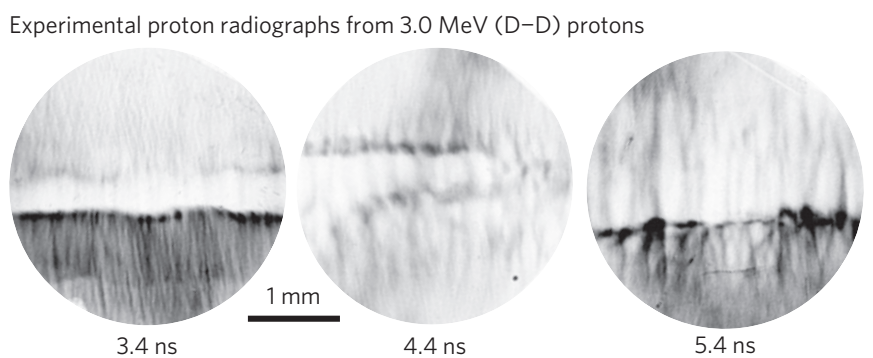

Experimental proton radiographs from $14.7 \mathrm{MeV}\left(\mathrm{D}-{ }^{3} \mathrm{He}\right)$ protons
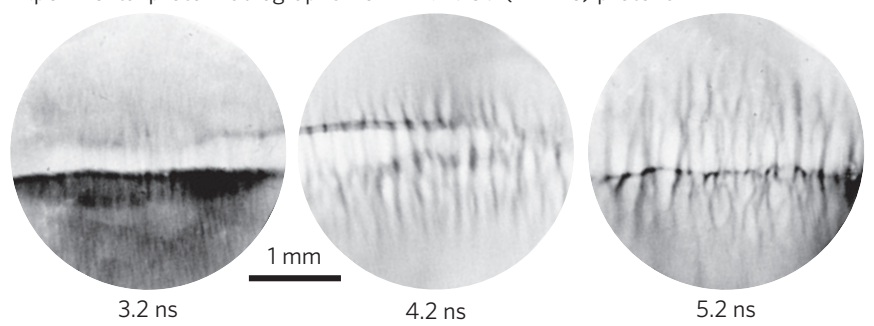

Synthetic proton radiographs from 14.7 MeV protons

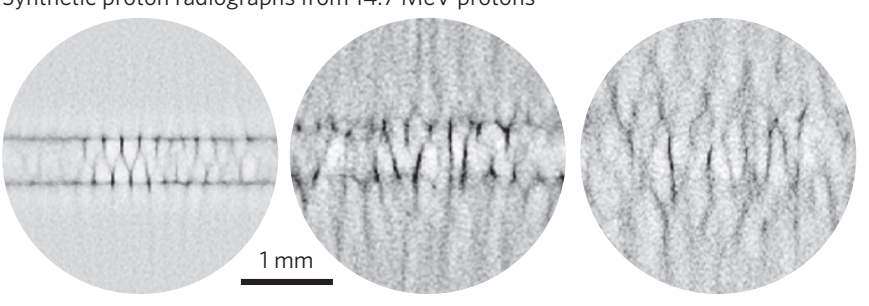

Figure 2 | Experimental images and synthetic radiographs of magnetic field structures. Experimental proton images are shown from 3.0 MeV D-D protons (top row), 14.7 MeV D- ${ }^{3} \mathrm{He}$ protons (middle row), and synthetic 14.7 MeV proton tracing from 3D particle-in-cell (PIC) simulations (bottom row). In each case the plasma flows enter the frame from the top and bottom. The small timing difference between D-D and D- ${ }^{3} \mathrm{He}$ images is due to the proton time of flight from the capsule to the plasma interaction region. At early time (approximately 3 ns after the drive begins), only initial traces of filamentation are observed. At later times the filaments become more coherent and increase in extent along the flow direction. In each case extended magnetic 'plates' are formed above and below the midplane as a result of the large-scale Biermann-battery fields generated in the laser-ablation process ${ }^{23}$. All images are $3 \mathrm{~mm}$ in diameter in the target plane.

the code OSIRIS (refs 26,27) to model, from first principles, the counter-streaming plasma flows and the generation of electromagnetic fields (Fig. 3a,b). The flows are initialized with the properties measured experimentally in the midplane region-namely, each flow has $n_{\mathrm{e}}=5 \times 10^{18} \mathrm{~cm}^{-3}, v=1,900 \mathrm{~km} \mathrm{~s}^{-1}$ and $T_{\mathrm{e}}=T_{\mathrm{i}}=1 \mathrm{keV}$. To include the effect of the Biermann battery, the flows were encircled by a large-scale magnetic field consistent with the misaligned density and temperature gradients of the flow, with an initial peak amplitude of $50 \mathrm{kG}$ (see ref. 19). Further simulation details are found in the Supplementary Information.

Within $1 \mathrm{~ns}$ of the opposing flows beginning to interact at the midpoint of the simulation volume, magnetic filaments are generated via the Weibel instability (Fig. 3b). Also, the magnitude of the toroidal Biermann-battery magnetic fields that are initially imposed doubles, owing to the conservation of magnetic flux. These fields lead to a long-range order in the system, and generate a pair of magnetic plates similar to those seen in the experiment (Fig. 2). The presence of the toroidal fields does not significantly alter the formation of the ion Weibel instability because the ions remain unmagnetized. This is supported by simulations where, when the 


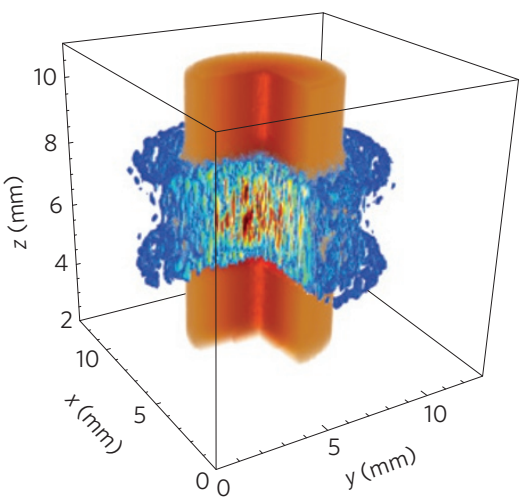

c

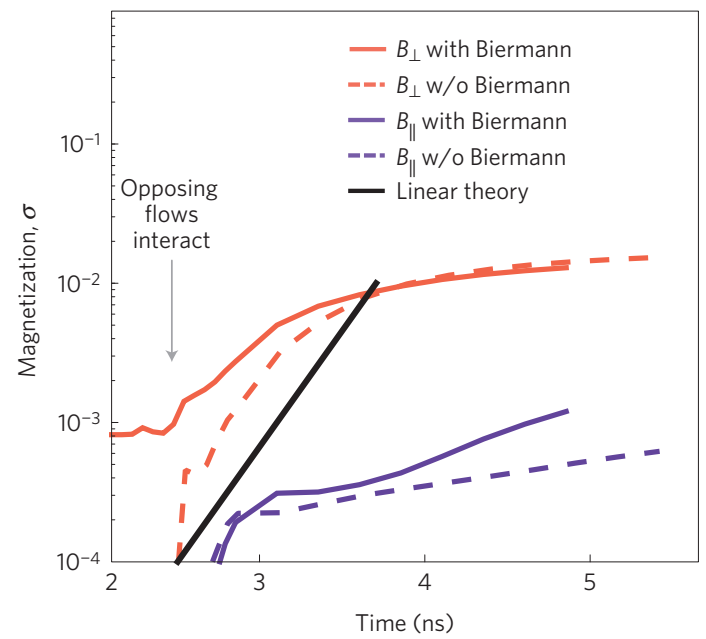

b

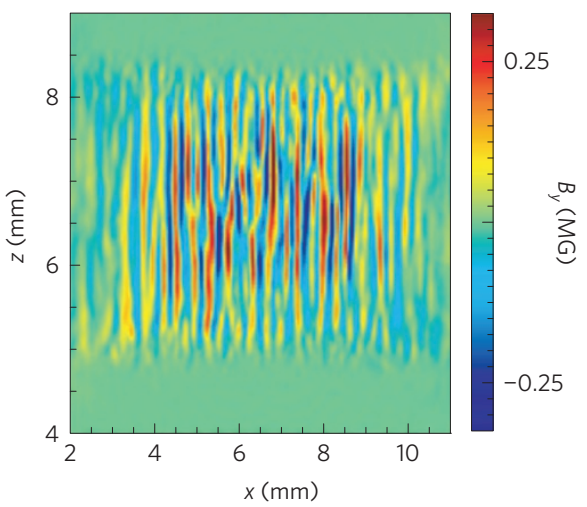

d

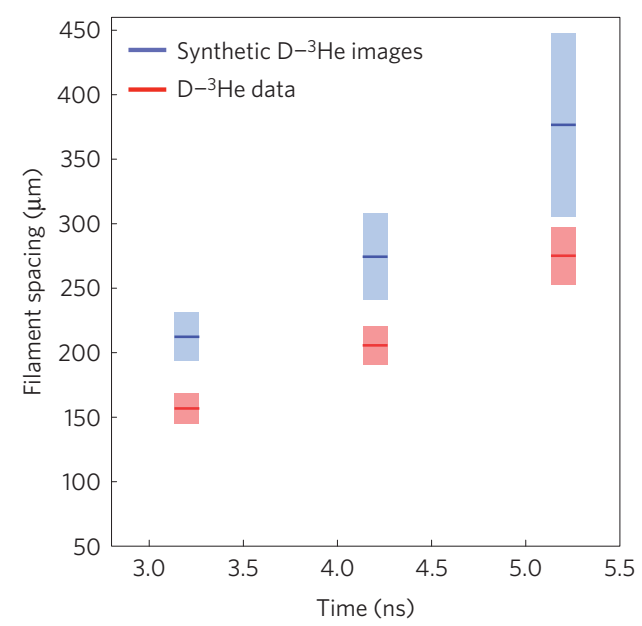

Figure 3 | Temporal evolution of magnetic field magnitude from simulation and field structure from experimental images. a, 3D OSIRIS simulation of the system after $1 \mathrm{~ns}$ of interaction between the counter-streaming 1,900 km s ${ }^{-1}$ plasma flows (approximately 3 ns after the experimental drive laser pulse; flows enter from top and bottom). Magnetic fields are shown qualitatively in the blue/red colour scale, with electron density in orange. $\mathbf{b}$, Magnetic field slice (transverse magnetic field component $B_{y}$ ) along the $y$-axis midplane, at the same time, illustrating the presence of strong filaments associated with the Weibel instability. c, Plasma magnetization, $\sigma$, as a function of time. When the flows are initiated with zero initial magnetic field (dashed lines) the magnetizations remain at zero until the flows begin interacting, between 2 and 3 ns. When initial toroidal fields are included consistent with the

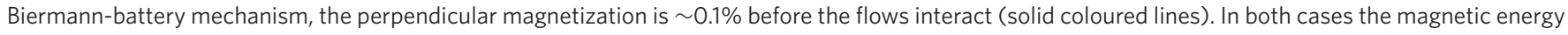
associated with Weibel instability increases sharply after the flows interact, increasing $\sigma$ by a factor greater than ten in several ns. The magnetization due to the ion Weibel instability, growing at the theoretical linear growth rate, is shown in solid black. This calculation shows that the Weibel-generated magnetization becomes the dominant contribution to the overall magnetization of the system. d, Measurement of the mean separation between filaments in experimental proton radiographs (red) and synthetic proton images from 3D PIC simulations (blue). The filament spacing approximately doubles over the 2 ns of observation. Note that time is experimental time, measured with respect to the beginning of the drive laser.

initial magnetic fields are not included, Weibel filaments are still produced with the same structure.

To properly compare the PIC results with the experimental radiographs, we have simulated the proton imaging directly in the 3D OSIRIS simulations to generate proton images of the electric and magnetic fields in the system. We assume an isotropic point source of $14.7 \mathrm{MeV}$ protons located $1 \mathrm{~cm}$ from the centre of the simulation box. The diagnostic protons interact with the $3 \mathrm{D}$ field structure during the simulation, and are then ballistically propagated to a $13 \mathrm{~cm} \times 13 \mathrm{~cm}$ detector plane $30 \mathrm{~cm}$ from the source, matched to the imaging system in the experiment.

The simulated proton radiographs are shown on the bottom row of Fig. 2, at the same times as the experimental data. To quantify the evolving structure in the system the filament spacing was measured for all images. Shown in Fig. 3d, the growth in the size of the Weibel features is seen to be nearly equal for the measured and simulated images. The increasing filament size indicates growing Weibel fields, and the efficiency of the instability to convert system kinetic energy into magnetic energy can be assessed directly from the simulations. The magnetic energy associated with the instability is driven by the ion flows, and goes mainly into the transverse component of the field. The amplitude of the Weibel magnetic fields grows exponentially during the linear phase, with a growth rate of $\sim 0.2 v / c \times \omega_{\mathrm{pi}}$ (where $c$ is the speed of light and $\omega_{\mathrm{pi}}$ is the ion plasma frequency), which is consistent with the linear theory of the instability. The linear phase of the instability saturates after $1-1.5 \mathrm{~ns}$ of interaction between the flows (that is, after $\sim 2-3$ e-foldings), although the field amplitude and filament size continue to increase in the subsequent nonlinear phase. The significance of the magnetic energy in the system is quantified by the magnetization parameter $\sigma$, defined as $\sigma \simeq\left\langle B^{2}\right\rangle / 4 \pi m_{\mathrm{i}} n_{\mathrm{i}} v^{2}$, where the spatially averaged field is given by $\left\langle B^{2}\right\rangle$, with $m_{\mathrm{i}}$ and $n_{\mathrm{i}}$ representing the ion mass and density, respectively. This ratio of magnetic energy to flow kinetic energy reaches nearly $1 \%$ by the end of the experimental interaction time (approximately $5 \mathrm{~ns}$ in Fig. 3c).

At times later than those probed in the experiment, the local magnetic field strength peaks at $0.6 \mathrm{MG}$, which corresponds to $\sigma=5 \%$. These high values illustrate the efficiency of the Weibel 
instability in converting kinetic energy into electromagnetic energy. At this amplitude the magnetic fields are large enough to cause the deflection of the incoming flows and the randomization of their kinetic energy. Furthermore, Weibel-mediated collisionless shocks are believed to occur at this level of magnetization, provided that there is a sufficiently large interpenetration distance-of the order of $300 \mathrm{c} / \omega_{\mathrm{pi}}$ (ref. 9). This condition precludes shock formation in the present experiment, where this length is only $55 \mathrm{c} / \omega_{\mathrm{pi}}$; however, a shock should be observed with a similar set-up if higher densities and/or longer flows are generated.

The dimensionless magnetization parameter $\sigma$ serves to connect experiments to astrophysical systems, and can be directly applied to scaled systems of interest ${ }^{16}$. For example, multiwavelength observations of afterglow emission of GRBs suggest sub-equipartition levels of magnetic field in the region behind the forward shock $(\sigma \approx 0.01-0.1$; ref. 28$)$. Such fields are probably generated near a collisionless shock front, as the pre-shock interstellar medium is effectively unmagnetized $\left(\sigma \approx 10^{-10}\right)$. Our experiments imply that even non-relativistic Weibel instability in an initially unmagnetized medium is capable of generating the percent-level magnetization observed in GRBs; collisions of relativistic flows are likely to produce even stronger fields.

Received 16 October 2013; accepted 7 November 2014; published online 19 January 2015

\section{References}

1. Bernet, M. L., Miniati, F., Lilly, S. J., Kronberg, P. P. \& Dessauges-Zavadsky, M. Strong magnetic fields in normal galaxies at high redshift. Nature 454, 302-304 (2008)

2. Medvedev, M. V., Silva, L. O., Fiore, M., Fonseca, R. A. \& Mori, W. B. Generation of magnetic fields in cosmological shocks. J. Korean Astron. Soc. 37, 533-541 (2004).

3. Medvedev, M. V. \& Loeb, A. Generation of magnetic fields in the relativistic shock of gamma-ray burst sources. Astrophys. J. 526, 697-706 (1999).

4. Gruzinov, A. \& Waxman, E. Gamma-ray burst afterglow: Polarization and analytic light curves. Astrophys. J. 511, 852-861 (1999)

5. Weibel, E. S. Spontaneously growing transverse waves in a plasma due to an anisotropic velocity distribution. Phys. Rev. Lett. 2, 83-84 (1959).

6. Moiseev, S. S. \& Sagdeev, R. Z. Collisionless shock waves in a plasma in a weak magnetic field. J. Nucl. Energy C 5, 43-47 (1963).

7. Lazar, M., Schlickeiser, R., Wielebinski, R. \& Poedts, S. Cosmological effects of Weibel-type instabilities. Astrophys. J. 693, 1133-1141 (2009).

8. Medvedev, M. V. Electron acceleration in relativistic gamma-ray burst shocks Astrophys. J. Lett. 651, L9-L11 (2006).

9. Kato, T. N. \& Takabe, H. Nonrelativistic collisionless shocks in unmagnetized electron-ion plasmas. Astrophys. J. Lett. 681, L93-L96 (2008).

10. Spitkovsky, A. On the structure of relativistic collisionless shocks in electron-ion plasmas. Astrophys. J. Lett. 673, L39-L42 (2008).

11. Martins, S. F., Fonseca, R. A., Silva, L. O. \& Mori, W. B. Ion dynamics and acceleration in relativistic shocks. Astrophys. J. Lett. 695, L189-L193 (2009).

12. Spitkovsky, A. Particle acceleration in relativistic collisionless shocks: Fermi process at last? Astrophys. J. Lett. 682, L5-L8 (2008).

13. Ryutov, D. D., Fiuza, F., Huntington, C. M., Ross, J. S. \& Park, H-S. Collisional effects in the ion Weibel instability for two counter-propagating plasma streams. Phys. Plasmas 21, 032701 (2014).

14. Kugland, N. L. et al. Self-organized electromagnetic field structures in laser-produced counter-streaming plasmas. Nature Phys. 8, 809-812 (2012).

15. Fox, W. et al. Filamentation instability of counterstreaming laser-driven plasmas. Phys. Rev. Lett. 111, 225002 (2013).
16. Ryutov, D. D. et al. Basic scalings for collisionless-shock experiments in a plasma without pre-imposed magnetic field. Plasma Phys. Control. Fusion 54, 105021 (2012).

17. Boehly, T. R. et al. The upgrade to the Omega laser system. Rev. Sci. Instrum. 66, 508-510 (1995).

18. Ross, J. S. et al. Characterizing counter-streaming interpenetrating plasmas relevant to astrophysical collisionless shocks. Phys. Plasmas 19, 056501 (2012)

19. Kugland, N. L. et al. Visualizing electromagnetic fields in laser-produced counter-streaming plasma experiments for collisionless shock laboratory astrophysics. Phys. Plasmas 20, 056313 (2013).

20. Ross, J. S. et al. Collisionless coupling of ion and electron temperatures in counterstreaming plasma flows. Phys. Rev. Lett. 110, 145005 (2013).

21. Takabe, H. et al. High-mach number collisionless shock and photo-ionized non-LTE plasma for laboratory astrophysics with intense lasers. Plasma Phys. Control. Fusion 50, 124057 (2008).

22. Kato, T. N. \& Takabe, H. Electrostatic and electromagnetic instabilities associated with electrostatic shocks: Two-dimensional particle-in-cell simulation. Phys. Plasmas 17, 032114 (2010).

23. Ryutov, D. D. et al. Magnetic field advection in two interpenetrating plasma streams. Phys. Plasmas 20, 032703 (2013)

24. Gregori, G. et al. Generation of scaled protogalactic seed magnetic fields in laser-produced shock waves. Nature Lett. 481, 480-483 (2012).

25. Schoeffler, K. M., Loureiro, N. F., Fonseca, R. A. \& Silva, L. O. Magnetic-field generation and amplification in an expanding plasma. Phys. Rev. Lett. 112, 175001 (2014)

26. Fonseca, R. et al. in Computational Science, ICCS 2002 Vol. 2331 (eds Sloot, P., Hoekstra, A., Tan, C. \& Dongarra, J.) 342-351 (Springer, 2002).

27. Fonseca, R. A. et al. One-to-one direct modeling of experiments and astrophysical scenarios: Pushing the envelope on kinetic plasma simulations Plasma Phys. Control. Fusion 50, 124034 (2008)

28. Frail, D. A., Waxman, E. \& Kulkarni, S. R. A 450 day light curve of the radio afterglow of GRB 970508: Fireball calorimetry. Astrophys. J. 537, 191-204 (2000)

\section{Acknowledgements}

We thank the staff of the Omega Laser Facility for their experimental support. This work was performed under the auspices of the US Department of Energy by the Lawrence Livermore National Laboratory, under Contract No. DE-AC52-07NA27344, with funding support from LLNL LDRD grant No. 11-ERD-054 and from the European Research Council under the European Community's Seventh Framework Programme (FP7/2007-2013), ERC grant agreement no. 256973. Computing support for this work came from ALCC and INCITE awards on Mira (ALCF supported under contract DE-AC02-06CH11357) and from the LLNL Institutional Computing Grand Challenge program on Vulcan. Additionally, the authors would like to acknowledge the OSIRIS Consortium, consisting of UCLA and IST (Lisbon, Portugal) for the use of the OSIRIS 2.0 framework and the visXD framework. F.F. acknowledges the LLNL Lawrence Fellowship for financial support. A.S. is supported by DOE grant DE-NA0002200.

\section{Author contributions}

This experimental platform was conceived by G.G., B.A.R., R.P.D., H.-S.P., D.H.F., Y.S., A.S. and H.T. It was developed in a series of initial experiments by N.L.K., C.C.K., J.M., T.M., C.P., J.S.R. and C.M.H., and the data shown here were collected in experiments by C.M.H., J.S.R. and H.-S.P. Theoretical support for this work was provided primarily by F.F. and D.D.R., as well as by A.S. and G.G. Support for proton diagnostics was provided by C.K.L., R.P., and particularly A.B.Z. Particle-in-cell simulations were performed and synthetic radiographs produced by F.F. Additional contributions to the analysis of proton radiographs by H.-S.P., J.S.R., M.C.L., A.B.Z., D.D.R., A.S. and C.M.H. The manuscript was written by C.M.H., F.F., J.S.R. and D.D.R.

\section{Additional information}

Supplementary information is available in the online version of the paper. Reprints and permissions information is available online at www.nature.com/reprints.

Correspondence and requests for materials should be addressed to C.M.H.

\section{Competing financial interests}

The authors declare no competing financial interests. 